\title{
Aspectos Didácticos En La Enseñanza De Conceptos Geobotánicos
}

\author{
Cano Ortiz Ana. ${ }^{1}$, Piñar Fuentes Jose Carlos ${ }^{1}$, Ighbareyeh J.M.H. ${ }^{2}$, Quinto Canas Ricardo ${ }^{3}$, \\ Cano Eusebio $^{1^{*}}$ \\ ${ }^{1}$ Department. of Animal and Plant Biology and Ecology, Section of Botany, University of Jaén, Campus \\ Universitario Las Lagunillas s/n, 23071 Jaén, Spain \\ ${ }^{2}$ Department of Plant Production and Protection, Faculty of Agriculture, Al-Quds Open University, Abu \\ Khatallah Street, Hebron, Hebron, Palestine \\ ${ }^{3}$ Faculty of Sciences and Technology, University of Algarve, Campus de Gambelas, 8005-139 Faro, Portugal; \\ Centre of Marine Sciences (CCMAR), University of Algarve, Campus de Gambelas, 8005-139 Faro, Portugal \\ *Corresponding Author: Cano Eusebio, Department. of Animal and Plant Biology and Ecology, Section \\ of Botany, University of Jaén, Campus Universitario Las Lagunillas s/n, 23071 Jaén, Spain
}

\begin{abstract}
Se tratan conceptos y métodos de estudio de la vegetación, proponemos métodos didácticos para estudiantes y jóvenes investigadores que quieran iniciarse en Ciencias de la Vegetación. Con este texto pretendemos visibilizar los últimos avances científicos en vegetación, proponemos los diversos métodos de estudio. Ponemos de manifiesto la importancia del trabajo de campo frente a los contenidos teóricos. Se explican algunos conceptos fundamentales, tales como la asociación vegetal y el concepto de serie de vegetación (sigmetum). Conceptos que son fundamentales para que el alumno pueda conocer e interpretar el paisaje, así como para hacer ordenaciones territoriales de carácter agrícola y forestal.
\end{abstract}

Key words: Vegetación, Asociación, Series, Dinámica, Fitoociología, Sintaxon, Sigmetum

\section{INTRODUCCIÓN}

A pesar de que en la Antigüedad se hicieron estudios relacionados con las plantas y con el ambiente por Aristóteles y Teofrasto, incluso se realizaron estudios de valor geobotánicopor Avicena y Alberto Magno, no es hasta el siglo XVIII cuando se consolidó una base científica, lo que permitido hablar en términos de Geobotánica.

En los tratados de botánicos antiguos, los de Teofrasto discípulo de Aristóteles, se encuentran observaciones fitoecológicas. En su obra "De causisPlantarum" se expone el principio de que cada especie es afectada por los factores medioambientales, a la vez que resalta la importancia de la exposición y la estratificación para comprender la distribución de los tipos de vegetación.

En el imperio romano cabe destacar la aportación de Plinio que aunque se ocupa esencialmente de plantas cultivadas, realiza numerosos comentarios biogeográficos sobre el paisaje vegetal en su obra "Naturalis Historia". Con la invasión de los bárbaros, se entra en un largo período de recesión en lo que se refiere al conocimiento y uso de las plantas, coincidiendo con un auge de la ganadería que pasa a ser la actividad económica principal.

En el periodo de esplendor cultural correspondiente a la dominación árabe en la península Ibérica, las ideas llegan desde Oriente, y aunque predominan los aspectos aplicados, pueden entresacarse algunas consideraciones fitogeográficas, de las numerosas obras de agricultura que se publican entonces. En España Avicena y Averroes parecen ser los primeros en expresar la idea de hábitat, así As Annabeti describe como los vegetales se asocian en comunidades específicas. La aportación de Edrisi es la más ambiciosa pues esboza la primera clasificación de los vegetales en función de las zonas climáticas. No obstante existieron otros autores que escribieron sobre el origen de las plantas cultivadas y sobre su aclimatación.

La vuelta a las ideas clásicas aristotélicas se realiza con Alberto Magno (1206-1280), valorándose las propiedades de las plantas según la naturaleza del lugar donde viven; ya en la obra de Alonso de 
Herrera, "Agricultura General" que se publica en 1513 en Alcalá de Henares, se hacen anotaciones corológicas y fitoecológicas sobre ciertas especies, así se dice que el boj nace en tierras frías y el algarrobo en lugares donde crecen las palmeras, es decir tierras cálidas. El verdadero precursor de la Geobotánica fue Alexander von Humboldt (1769-1859) que fue el primero en plantearse la descripción de los paisajes vegetales con una óptica eminentemente fitogeográfica.

Si bien a lo largo de la historia la Geobotánica ha venido utilizándose, al emitir conceptos y aspectos metodológicos por investigadores, no es hasta el siglo XX cuando adquiere un gran desarrollo con la aparición de diversas escuelas.

Flahault y Schroter pueden considerarse los precursores de la Sociología Vegetal o Fitosociología en centroeuropa, mientras Warming inspira la tradición anglosajona, los seguidores de Warming, investigando con puntos de vista netamente ecológicos, forman la denominada escuela anglonorteamericana con Clements en Estados Unidos y Tansley en Gran Bretaña, mientras que en el viejo continente se ponen los cimientos de la moderna Fitosociología (Ciencia de la Vegetación), por el contrario Rübel y Brockmann-Jerosch, se mantienen en las grandes visiones de abstracción como recomendara Humboldt, estableciendo las grandes formaciones de vegetación de la Tierra.

En el área mediterránea ha adquirido gran desarrollo la escuela sigmatista (S.I.G.M.A., StationeInternationaleMéditerranéenne et Alpina, Montpellier 1930), basada en el concepto de asociación y las técnicas muestreo propuestas por Braun-Blanquet en su obra Pflanzensociologie, que junto a Pavillard fundaron la escuela sigmatista, implantándose así la Sociología Vegetal (Fitosociología).

En la primera mitad del siglo XX tiene relevancia en España la obra de Huguet de Villar, el cual define la Geobotánica como "la Ciencia de la relación entre la vida vegetal y el medio terrestre" o "la Ciencia que estudia el hábitat de las plantas en la superficie terrestre". Ya en la segunda mitad del siglo XX Ehrendorfer la define como "la ciencia que estudia la distribución de las plantas en la superficie de la Tierra y trata de conocer las características esenciales de la misma, las leyes a que obedece y las causas a que es debida. O como el mismo autor aclara años más tarde: Es objeto de la Geobotánica, reconocer la distribución y la vida en común de las estirpes vegetales, apreciar los rasgos generales y las reglas que presiden estos hechos y esclarecer las causas de los mismos." A partir de esta segunda mitad del siglo XX se dispara el estudio y la investigación del medio natural, y en concreto de la vegetación. Se establecen dos líneas de investigación claramente diferenciadas, la netamente ecológica por la escuela anglonorteaméricana, que se implanta en Reino Unido y en Norteamérica, y la escuela centroeuropea que se desarrolla ampliamente en Europa irradiando a norte de África con investigadores franceses y españoles, y a Centroamérica. Si bien ambas escuelas tienen el mismo objetivo, el conocimiento de la vegetación, utilizan metodologías diferentes, siendo diferentes las técnicas de muestreo.

Si bien el conocimiento de las especies ha sido y sigue siendo fundamental para el desarrollo ulterior de la Fitosociología, no menos importante es el fuerte avance, que de la mano del profesor Rivas Martínez ha tenido la Bioclimatología, como base esencial en la descripción de las fitocenosis, así como en las planificaciones agrícolas, forestales y ganaderas[1], [2], [3], [4], [5].

La Bioclimatología es una ciencia ecológica, que ha adquirido importancia en los últimos años y que trata de poner de manifiesto la relación existente entre los seres vivos (Biología) y el clima (Física). Se diferencia de la Climatología en que la información, índices y unidades que utiliza están relacionados y delimitados por especies y fitocenosis (biocenosis). El desarrollo de la Bioclimatología como disciplina básica al servicio de la Geobotánica ha sido uno de los aspectos científicos más sobresalientes en los últimos tiempos; el progreso de esta ciencia ha permitido diagnosticar mejor muchas comunidades vegetales, y sobre todo poder precisar mejor las principales cesuras que se observan en la cliserie altitudinal.

Para el estudio de la Geobotánica y para las planificaciones agrícolas, ganaderas y forestales, es importante conocer los aspectos bioclimáticos y biogeográficos. De los diferentes factores que conllevan a la existencia de determinados ecosistemas vegetales, la precipitación y la temperatura son de los más importantes. Así cada región o grupo de regiones biogeográficas, posee una peculiar zonación altitudinal de los ecosistemas vegetales; tal cliserie o series concatenadas, se debe al progresivo descenso de la temperatura media anual con la altitud (termoclima).Si se correlaciona el clima (temperatura y precipitación) con las discontinuidades biocenóticas que aparecen en las 
montañas con la altitud (cliseries altitudinales), veremos que se cumplen en toda la tierra ciertos ritmos o cambios en función de la $\mathrm{t}^{\mathrm{a}} \mathrm{y}$ la precipitación (termoclima y ombroclima). En consecuencia, en función de tales cambios se puede reconocer por un lado el continente físico, que son los pisos bioclimáticos y por otro el contenido biológico vegetal que son las series de vegetación.

\section{Materiales Y Métodos}

Desde el punto de vista metodológico es importante que el estudiante aprenda las distintas técnicas de muestreo de la vegetación. Debiendo obtener una capacidad de observación para actuar de forma autónoma, para ello es preciso el desarrollo de sus capacidades cognitivas.

\subsection{Método fitosociológico}

Esté método consta de dos fases, la primera de carácter analítica tiene una gran importancia, pues debe hacerse de forma minuciosa la selección de las parcelas de muestreo, la segunda fase será de tipo sintético. Con este método se seleccionan parcelas homogéneas y se toma la cobertura total de la fitocenosis. El grado de cobertura se da en $\%$ y representa la superficie de suelo cubierta de vegetación. Para calcular el porcentaje de cobertura se mide la parcela muestreada que tendrá un valor variable dependiendo del tipo de fitocenosis, del bioma en el que está integrada la comunidad vegetal, por ejemplo en áreas mediterráneas el área de muestreo para los bosques suele oscilar entre 300-500 $\mathrm{m}^{2}$, mientras que en zonas tropicales y subtropicales este área debe incrementarse aproximadamente a $1000-2000 \mathrm{~m}^{2}$, para las comunidades de matorral se toman entre $50-200 \mathrm{~m}^{2}$, los pastizales entre $0,5-2$ $\mathrm{m}^{2}$., en todos los casos es conveniente realizar el cálculo del área mínima para obtener la máxima diversidad florística en la mínima superficie. Si una parcela tiene $100 \mathrm{~m}^{2}$ y hay cubiertos $45 \mathrm{~m}^{2}$, significa que el grado de cobertura es del $45 \%$.

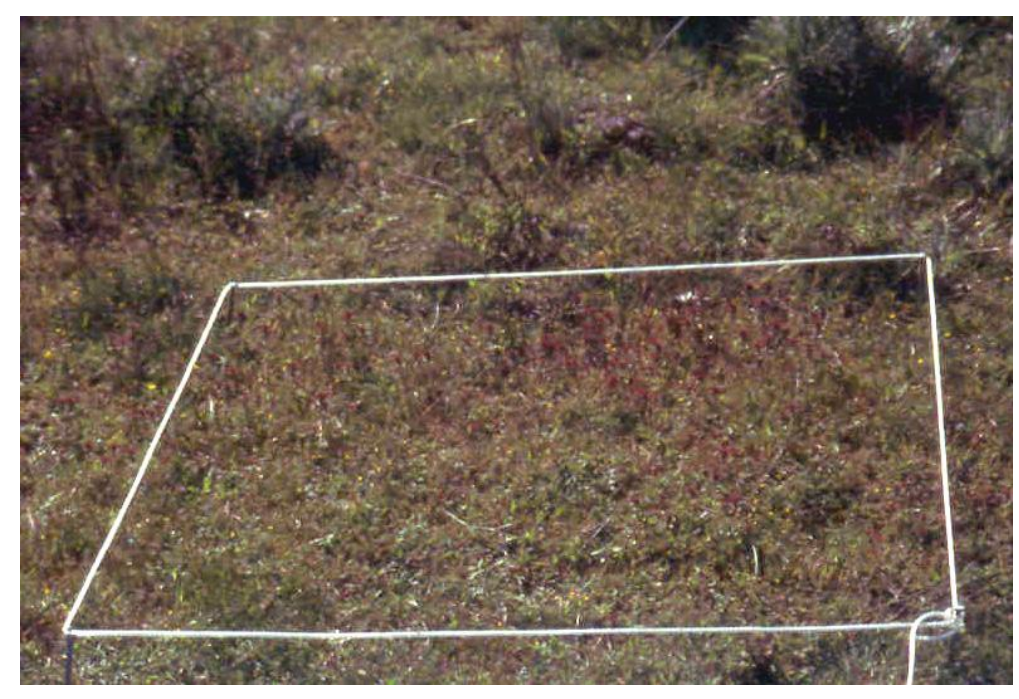

Figura1. Cálculo del área mínima de una comunidad vegetal de pastizal

A continuación se hace una enumeración del número de especies y se extrae la cantidad total de especies (estima global), siendo esta estima global un factor indicador de la biodiversidad.

A cada especie se le da un índice de abundancia-dominancia, que índica el número de individuos de una determinada especie, estos índices son: $+, 1,2,3,4,5$.

+ indica individuo raro en la parcela seleccionada, este índice se da cuando aparece 1 o 2 pies de planta.

1 indica individuos muy escasos, se da este índice cuando existen algunos pies de plantas aislados.

2 indica que son individuos escasos

3 poco abundante, pero en este caso la planta cubre ya parte del suelo

4 planta abundante

5 planta muy abundante

Los índices del + al 2 presentan grado de cobertura pequeño inferior al 50\%, el 3 se debe corresponder con cobertura que oscila entre el $55-75 \%$, el 4 con $75-90 \%$ y el 5 del $90-100 \%$ [6]. 


\section{RESUltAdOS Y DiscuSióN}

Como resultado de la aplicación del método fitosociológico,se diagnostica una determinada comunidad vegetal, de lo que se ocupa la sintaxonomía, para ello es necesario respetar las normas internacionales, establecidas en el Código de Nomenclatura Fitosociológica[7], código en el que se establece un sistema de rangos sintaxonómicos, siendo la asociación la unidad básica del sistema.

La sintaxonomía debe entenderse como la sistemática de las comunidades vegetales, donde existe un sistema de rangos jerárquicos de clasificación que se reconocen para una fitocenosis. La unidad básica de la sintaxonomía es la asociación vegetal. En la clasificación existen diferentes unidades sintaxonómicas de vegetación, las cuales se establecen en base a la presencia o ausencia de especies botánicas. Estas unidades son la subasociación, asociación, alianza, orden y clase.

La asociación es la unidad básica fitosociológica que se corresponde a lo que es la especie en la sistemática de los táxones, siendo esta la unidad fundamental de la fitosociología, que está basada en los inventarios de vegetación, realizados en condiciones homogéneas ecológicamente, las asociaciones deben tener una composición florística propia y original, estadísticamente fieles a su distribución biogeográfica y a su hábitat, estando subordinada a la asociación la subasociación y la variante. Una fitocenosis concreta para ser considerada asociación debe presentar un mínimo de especies que la caracterizan y de compañeras. La especies características junto con otras que aparecen de forma constante, que son propias de rangos sintaxonómicos superiores, constituyen el armazón básico de la comunidad; cuanto mayor sea el número de especies constantes con respecto al número total, más homogénea es la asociación y cuanto más alta sea la proporción de especies características, mejor caracterizada estará la asociación desde el punto de vista florístico y ecológico. Puede ocurrir que la asociación como tal se encuentre fragmentada, bien porque falta espacio, hábitat desfavorable, intervenciones humanas, animales. Se entiende por especie característica aquella que se presenta en una comunidad con un grado de presencia y caracteriza a la comunidad desde el punto de vista florístico, ecológico y corológico. Para que una planta caracterice a una asociación debe presentar un área mínima de distribución, ya que si dicha área es excesivamente grande esta planta pasa a caracterizar a sintaxones de rango superior y si su área es inferior al mínimo de la asociación pasa a caracterizar a la subasociación. Por ello en toda fitocenosis pueden existir taxones que sean característicos de subasociación, asociación, alianza, orden y clase; esto va a estar en relación directa con la estenoicidad de la planta, a mayor estenoicidad menor área de distribución, por ello en este caso caracteriza a sintaxones de rango inferior y viceversa [8].

Existen además otras especies que no caracterizan la comunidad y que se les llama compañeras, se trata de especies de otras asociaciones vecinas que por su estenoicidad no demasiado acusada se introgreden en la asociación de estudio, estas plantas son introgresivas, de forma similar aquellas especies de la asociación de estudio pueden transgredir a las comunidades vecinas, se trata en este caso de especies transgresivas, se habla de especies diferenciales cuando son plantas que existen en una comunidad (asociación) y provocan una diferenciación respecto de la asociación tipo, esto significa que cuando se describe una comunidad con una determinada composición florística, si en estudios posteriores de esta comunidad aparecen plantas diferentes de la diagnosis original, se habla de especies diferenciales.

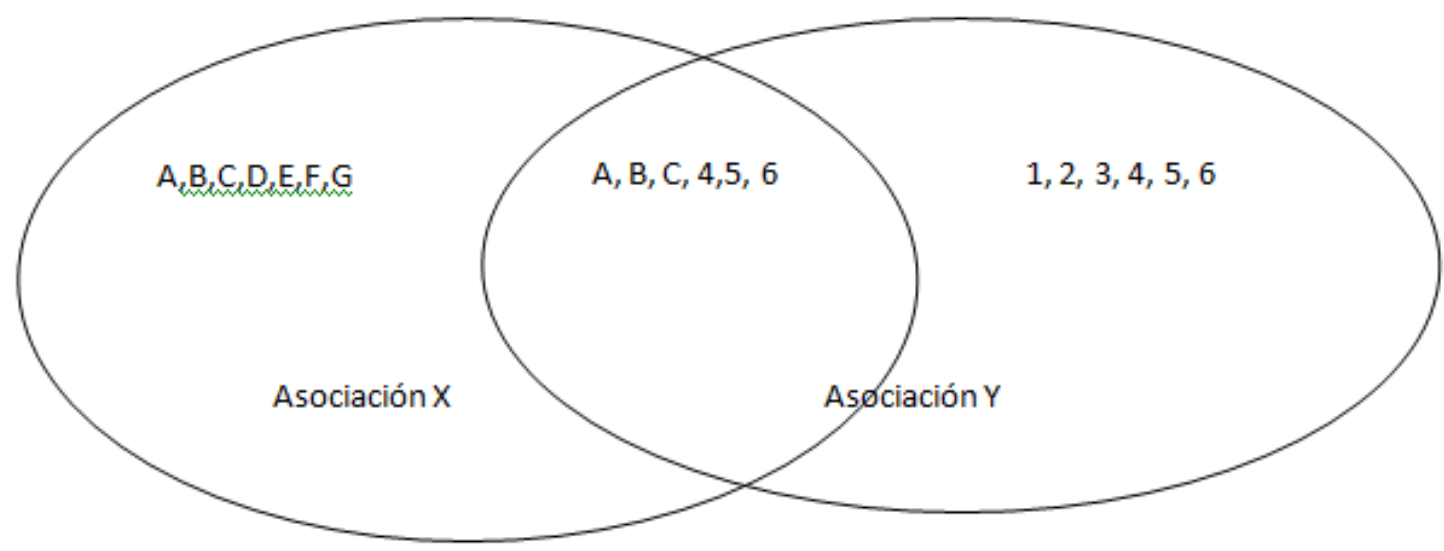

Figura2. Especies diferenciales y comunes a las asociaciones $x, y$ 
Teniendo en cuanta dos asociaciones en vecindad, la asociación $\mathrm{x}$ tiene como plantas características A,B,C,D,E,F,G y la asociación y tiene como especies que la caracteriza 1, 2, 3,4,5 y 6 . La zona de contacto se conoce como ecotono y encierra especies de la asociación x $(\mathrm{A}, \mathrm{B}, \mathrm{C})$ y especies de la asociación y $(4,5,6)$. El ecotono es una zona intermedia que aparece por el cambio gradual de los factores ecológicos, siendo esta un área rica en plantas, ya que coexisten especies de las dos asociaciones, este caso intermedio se trata como variante ecológica de carácter edáfico, climático etc, sólo en el caso de que existan plantas que además del matiz ecológico presenten un matiz corológico y dinámico se habla de especies diferenciales, que sirven para diagnosticar a una subasociación de la asociación.

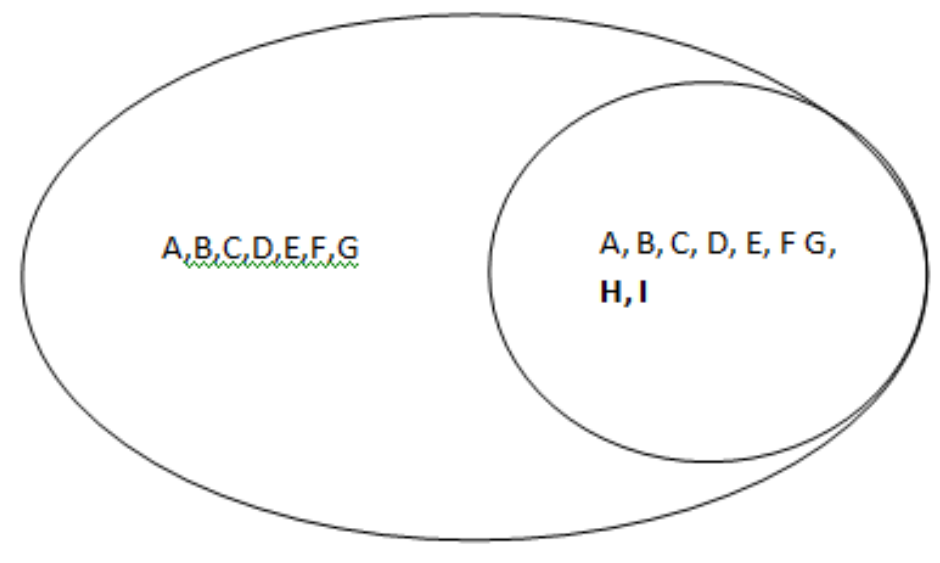

Asociación X. Subasociación

Figura3. Diferenciales de subasociación respecto a la asociación $x$

En este caso las especies H, I actúan como diferenciales de la asociación original, por lo que caracterizan a una subasociación L.

De forma similar a lo que ocurre con la asociación, los criterios para diagnosticar los sintaxones de rango superior: alianza, orden y clase, se basan en el principio de fidelidad, por ello lo mismo que se habla de especies diferenciales y características de asociación, existen también especies diferenciales y características de alianza, orden y clase. La alianza es la unidad inemediatamente superior a la asociación que reúne a las asociaciones vegetales semejantes, caracterizadas por especies características de alianza. Estas especies no se presentan o se presentan en baja cantidad en las demás alianzas, ya que su óptimo ecológico se encuentra en una determinada alianza, evidentemente existirán especies características de alianza, transgresivas, diferenciales, introgresivas. Pudiendo ocurrir que en una misma alianza se reúnan asociaciones muy dispares ecológicamente. La alianzas florísticamente próximas se reúnen en órdenes y los órdenes florísticamente semejantes se reúnen en clases de vegetación. Las especies características de clase tienen una gran amplitud ecológica, por tanto son menos estenoicas que las de los sintaxones inferiores, pudiendo servir las especies características de los órdenes, alianzas y asociaciones para caracterizar florísticamente a la clase que los incluye.

La Sinfitosociología es una ciencia absolutamente nueva, ya que si bien los aspectos conceptuales se conocían desde comienzos del siglo XX, han sido los fitosociólogos contemporáneos quienes han desarrollado científicamente las ideas de integración de las comununidades, como algo característico del paisaje. Destacamos en este campo a Braun-Blanquet, Tüxen, Bolós, Gèhu, Rivas-Martínez; ya que han sido los primeros en enunciar las bases filosóficas esenciales de esta nueva metodología para el estudio del paisaje [9].A partir de este momento muchos han sido los autores que han trabajdado con esta metodología[10],[11], [12]. El sigmetum, sinasociación o serie de vegetación existe como tal por la existencia de un clima territorial, por ello la serie de vegetación es de carácter climatófilo[13],[14], existiendo un proceso dinámico-catenal propio en cada serie de vegetación[15]. Las cabezas de series de vegetación representan la climax territorial, su composición florística depende del termoclina y del ombroclima, en este sentido los territorios pueden presentar bosques de lluvia [16], [17], [18], [19], [20], [21], [22]. En el caso de no depender del agua de lluvia, sino que el suelo es el factor determinante estamos ante una serie edafófila, pudiendo ser esta última de tipo edafohigrófilo (serie riparia) o bien edafoxerófilo (comunidades de roquedos) [12],[4],[5],[23],[24]. 


\section{CONClusión}

Al ofrecer al alumno los diferentes métodos para estudiar la vegetación, entre los cuales está el método ecológico de la escuela anglosajona y el fitosociológico de la escuela centroeuropea, y al aplicar dichos métodos en campo, el alumno llega a la conclusión, de la imposibilidad de aplicar con eficacia el método ecológico en ambientes mediterráneos, debido a la gran diversidad de hábitats que estos ambientes presentan. Lo que es lógico ya que el método ecológico fue ideado para ambientes templados, zonas homogéneas de baja diversidad florística. Sin embargo cuando el alumno utiliza el método fitosociológico descubre que este es operativo en ambos ambientes, tanto en zonas mediterráneas como en zonas centroeuropeas templadas e incluso en territorios subtropicales como tropicales, solo que en este último caso el tamaño de la parcela suele ser dos a tres veces superior que en áreas mediterráneas. Una vez asimilado este conocimiento metodológico, el alumno está preparado para la realización de muestreos y la interpretación y adjudicación de estos a una determinada asociación vegetal y a un sistema jerarquizado de rangos sintaxonómicos. En esta situación el alumno aprende a diferenciar unas comunidades vegetales de otras, y establece un sistema de clasificación. Conocidas las comunidades vegetales, el siguiente paso consiste en relacionar dichas comunidades y pasar a comprender lo que es un sigmetum o serie de vegetación.

\section{REFERENCIAS}

[1] Rivas-Martínez S. and Loidi Arregui J. Bioclimatoloy of theIberianPeninsula. ItineraGeobotanica, vol. 13, 41-47 (1999).

[2] Rivas-Martínez S., Díaz T. E., Fernández-González F., Izco J., Loidi J., Lousa M. and Penas A. Vascular PlantCommunities of Spain and Portugal. ItineraGeobotanica 15(1), 5-432 (2002a).

[3] Rivas-Martínez S., Díaz T. E., Fernández-González F., Izco J., Loidi J., Lousa M. and Penas A. Vascular PlantCommunities of Spain and Portugal. ItineraGeobotanica 15(2), 433-922 (2002b).

[4] Rivas-Martínez, S. Mapa de series, geoseries y geopermaseries de vegetación de España. Parte I. ItineraGeobotanica 17, 5-436 (2007).

[5] Rivas-Martínez S. and co-authors. Mapa de series, geoseries y geopermaseries de vegetación de España. Parte II. ItineraGeobotanica 18(2), 425-800 (2011).

[6] Braun-Blanquet J. (1979). Fitosociología, Ed. Blume, Madrid. Pp. 820 (1979)

[7] Theurillat J. P., Willner W., Fernández- González F., Bültmann H., Čarni A., Gigante D., Mucina L. and Weber H. International Code of Phytosociological Nomenclature. 4th edition. Applied Vegetation Sciene. Pp. 1-169 (2020) https://doi.org/10.1111/avsc.12491.

[8] Gehú J.M. and Rivas Martínez S. Notionsfondamentales de Phytosociologie. Syntaxonomie. J.Cramer. Vadeuz. (1981).

[9] Rivas Martínez S. Memoria del mapa de series de vegetación de España 1:400.000. Ministerio de Agricultura, Pesca y Alimentación. I.C.O.N.A. Pp. 268 (1987).

[10] Pinto Gomes C.J. Estudio Fitoassociológico do BarrocalAlgarvio (Taira, Portimao, Ph.D. Tesis Universidade de Évora. Portugal. Pp. 662 (1998)

Citation: Cano Eusebio, et.al. "Aspectos Didácticos En La Enseñanza De Conceptos Geobotánicos" International Journal of Humanities Social Sciences and Education (IJHSSE), vol 8, no. 4, 2021, pp. 271-276. doi: https://doi.org/10.20431/2349-0381.0804022.

Copyright: (C) 2021 Authors. This is an open-access article distributed under the terms of the Creative Commons Attribution License, which permits unrestricted use, distribution, and reproduction in any medium, provided the original author and source are credited. 